\title{
Direct Olive Oil Analysis
}

\author{
By M. Valcárcel`, M. Gallego, S. Cárdenas and F. Peña
}

\author{
Analytical Chemistry Division, University of Córdoba, \\ Edificio C-3 Anexo, Campus de Rabanales, 14071 Córdoba, Spain. \\ Phone and Fax: + 34957218616 \\ E-mail: qa1meobj@uco.es
}

\section{CONTENTS}

1. Introduction

2. Direct analysis of olive oil

3. Automated preparation of olive oil samples prior to analysis References

\section{RESUMEN}

\section{Análisis directo de aceite de oliva.}

El impacto práctico del análisis directo es tan innegable como que el contribuye decisivamente a mejorar las denominadas características analíticas relacionadas con la productividad como la rapidez, la reducción de costes y la minimización de riesgos para los analistas y el ambiente. El principal objetivo es establecer un adecuado "bypass" a las operaciones convencionales preliminares del proceso analítico. Este artículo ofrece una propuesta sistemática en este contexto y resalta el gran campo de acción de las metodologías directas en los análisis de rutina del aceite de oliva. Se analizan los dos tipos principales de metodologías. Por una lado, se analiza la determinación directa de los compuestos volátiles. Por el otro, se presentan y discuten los procedimientos simples para implementar automáticamente las operaciones preliminares del análisis del aceite usando sistemas simples en los que la muestra se introduce directamente con/sin una dilución simple.

PALABRAS-CLAVE: Aceite de oliva - Análisis directo - Automatización - Cromatografía - Espacio de cabeza -Espectrofotometría.

\section{SUMMARY}

\section{Direct olive oil analysis.}

The practical impact of "direct analysis" is undeniable as it strong contributes to enhance the so-called productive analytical features such as expeditiousness, reduction of costs and minimisation of risks for the analysts and environment. The main objective is to establish a reliable bypass to the conventional preliminary operations of the analytical process. This paper offers a systematic approach in this context and emphasises the great field of action of direct methodologies in the routine analysis of olive oil. Two main types of methodologies are considered. On the one hand, the direct determination of volatile components is systematically considered. On the other hand, simple procedures to automatically implement the preliminary operations of the oil analysis using simple devices in which the sample is directly introduced with/without a simple dilution are present and discussed.

KEY-WORDS: Automation - Direct analysis - Gas chromatography - Headspace - Olive oil - Spectrophotometry.

\section{INTRODUCTION}

In the field of food analysis, the development of laboratory methodologies providing rapid and reliable analytical information is of crucial importance with the view to making timely, well-found decisions to answer an economic or social problem. Current tendency is focused on obtaining general answers (based on binary yes/no response) rather than on detailed, discriminated information (Valcárcel et al. 1999). It aims at adapting proposed methodologies to ensure consistency between the chemical information delivered with that requested by the clients (Valcárcel et al. 2000). In this regards, analytical processes must be designed and applied to minimise or avoid the need for the preliminary steps required to make compatible the analyte(s) present in the sample with the instrument selected to carry out the measurement.

The automation, miniaturisation and simplification of the analytical processes are, therefore, main objectives in nowadays Analytical Chemistry as they are aimed at reducing or avoiding the human participation, minimising the size of the elements (devices, apparatus and instruments) used in the analytical process as well as at reducing the extension of the analytical method in whole or in any of its steps. Undoubtedly, they involve keywords (catchwords) that are used to attract potential users of the proposed methodologies and to describe their potential advantages when, in fact, only partial approaches are considered. One case in mind is the so-called "direct analysis" which has been increasingly used in the analytical chemical literature in the last few years. Close scrutiny of the papers that use it reveals the need to establish a clear definition of what is called "direct analysis".

A strict consideration of what "direct analysis" is, will let us include only those methodologies in which the analytical process acts as a black-box where the sample is directly introduced in the instrument (analyser) and it provides the results without the presence of the operator. In this context, only those determinations requiring no pretreatment (or only a dilution of the sample) will be considered as pure direct analysis. However, if the different degree of automation achieved for the three steps of an analytical process and the influence of the preliminary operations in the quality of the analytical results obtained on account of their negative 
connotations (Valcárcel et al. 1993) are considered, all those methodologies focused on developing reliable bypass to these preliminary operations (such as sampling, sample-size measurement, homogenisation, dissolution continuous separation techniques, derivatisation and transfer to the instrument) in order to reduce their adverse connotations can be included under "direct analysis".

The main aim of this paper is to offer an overview of the methodologies proposed for the direct analysis of olive oil on the basis of the previously established definition. As the monograph is devoted to emerging analytical techniques in the field of edible oils and fats, classical direct methods (mainly those based on volumetric determinations) would be out of the scope of this article.

\section{DIRECT ANALYSIS OF OLIVE OIL}

Direct analysis of olive oil refers almost exclusively to the determination of the volatile compounds present in the sample. It has been established the presence of more than 200 compounds in this fraction from which approximately 50 have been properly identified (Morales et al. 1994; Overton and Manura 1995). It is obvious that in these analyses, the requested analytical information is not a large list of compounds with their individual concentrations but the determination of the profile of such fraction for quality control, detection of contaminants in a pure product and determination of the origin of an olive oil.

These analyses can be carried out by means of three different methodologies, namely: (i) headspace with chromatographic separation (HS-GC); (ii) headspace without chromatographic separation (chemical sensor) and (iii) electronic olfactometry (e-noses). The main aspects of these alternatives as applied to the analysis of olive oil samples will be commented below.

The headspace technique is used for the direct determination of the volatile compounds present in an olive oil sample with chromatographic separation using different detectors or by direct introduction of the vapour phase into a mass spectrometer (chemical sensor). The former provides detailed information of the components present in such fraction while the later is focused on characterisation of the olive oil on the grounds of the monitored ions.

Concerning HS-GC, the existing methods vary in the way the analytes are introduced into the GC. In the most simple methodology developed, a small amount of olive oil (ca. $5 \mathrm{~g}$ ) is placed in a glass vial which is further sealed with a PTFE-coated silicon septum. The vial is then slightly heated to favour the analytes from the matrix passing to the headspace. After an equilibrium time, an aliquot of the gas phase is transferred to the injection port of a GC. Styrene
(Nerin et al. 1993) and tetrachloroethylene (Norman 1991) have been determined in olive oil by using a mass spectrometer or an electron capture detector, respectively. The determination of the volatile fraction present in a virgin olive oil usually involves the preconcentration of the analytes prior to introduction into the GC. One alternative requires the use of a purge and trap system (Morales et al. 1994; Overton and Manura 1995) where the volatiles are carried with an inert gas to a Tenax TA trap. The volatiles are then thermally desorbed (typically by heating at $220^{\circ} \mathrm{C}$ for $5 \mathrm{~min}$ ) and collected in a fused silica cold trap (ca. $-100^{\circ} \mathrm{C}$ ). Finally, the volatiles are transferred to the $\mathrm{GC}$ column by flash heating $\left(\mathrm{ca} .180^{\circ} \mathrm{C}\right.$ ) and detected by mass spectrometry. The other possibility involves the determination of the volatile fraction by stripping the analytes from the olive oil sample with a nitrogen stream at $37^{\circ} \mathrm{C}$ for $2 \mathrm{~h}$ and concentrating them onto a $50 \mathrm{mg}$ activated charcoal column; elution is carried out with $1 \mathrm{~mL}$ of diethyl ether. Once separated, the different organic compounds are detected by flame ionisation detector (FID) (Angerosa et al. 1996).

Mass spectrometers specifically configured for rapid headspace analysis were introduced in 1998 seeking to augment or even replace human sensory evaluation. This instrument, also called chemical sensor responds to all volatile compounds in the sample, providing structural information with minimal sample preparation (Meng and Wylie 1999). It also allows fast method development with a sample throughput of 15-30 $\mathrm{h}^{-1}$ (depending on the equilibration time required). In the field of food analysis, research is focused on the study of authenticity of edible oils and fats to detect potential frauds. As general procedure, the olive oil samples (typically $5-10 \mathrm{~mL}$ ) are placed in glass vials sealed with crimp caps and PTFE-coated silicone rubber septa. After the vials are heated (and/or shaken) for a predefined period of time (ca. $30 \mathrm{~min}$ ), $1 \mathrm{~mL}$ of the headspace volatiles are transferred, by means of a six-port high pressure injection valve, directly to the mass selective detector. As there is virtually no separation of the volatile constituents, the signal obtained is a single, broad peak composed of all the volatile constituents in the olive oil sample. After a training step, the instrument is able to classify unknown samples using multivariate analysis and a dedicated pattern recognition software. In this way, similar samples cluster together on the plot and samples that differ in their volatile components (because of composition, grade, impurity, manufacturing process, etc) cluster in different locations. This hyphenated technology have been sometimes considered as an electronic-nose, being however, remarkable differences between them.

The electronic olfactometry employs a series of discrete solid chemical and electrochemical sensors 
with partial specificity for the measurement of volatiles. The interaction of the volatiles is usually carried out by physical or chemical adsorption and desorption or reactions that take place on the surface of the sensor or in the bulk of the material which the sensor is made of. These interactions cause changes in physical properties (conductivity, direct polarisation, optical properties, magnetic and dielectric properties, etc.) which are recorder against the time. These data are further treated by complicated mathematical algorithms to obtain a pattern or correlation model between them for data interpretation (García-Pinto et al. 2001). The response generated is basically a chemical fingerprint of the sample that allows its identification even in complex mixtures. The perfect sensor for each kind of sample does not exist, but most olive oil applications employ conducting polymers as sensors. These are a specific kind with the property of working at room temperature that respond to a broad range of vapours (Gardner and Bartlett 1999). They are obtained from monomers (mainly pyrrol, aniline, thiophene and indol) by electropolimerisation with different counterions. The sensitivity and selectivity of these sensors are achieved with the variation on functional groups, the structure of the polymer and using different ions (García-Pinto et al. 2001). The main shortcoming of an e-nose instrument is the risk of sensor poisoning by humidity and the presence of alcohol, two situations which could affect or even contribute to the produced signal. Most applications described about olive oil refer to its characterisation (Stella et al. 2000) and quality control of the aroma of the virgin olive oil (Guadarrama et al. 2000). The instrument is described elsewhere in this volume.

\section{AUTOMATED PREPARATION OF OLIVE OIL SAMPLES PRIOR TO ANALYSIS}

Continuous flow systems in general and flow injection $(\mathrm{FI})$ in particular are very useful methodologies for automatic direct analysis of aqueous samples; the analysis of complex matrixes (e.g. soils, food, biological fluids, etc.) usually involve a sample pre-treatment step before introduction into the continuous systems. Nevertheless, the nature of some samples require the use of organic solvents, being the determinations of characteristic parameters in these samples difficult to automate. Fats and oils are clear examples in this category. Oil samples should be properly diluted with an organic solvent prior to introduction into the flow reaction system in order to reduce the high viscosity of the matrix, avoid emulsification and, hence, irreproducibility. Choosing an appropriate organic diluent is crucial for correct performance of the method. Direct analysis of olive oils have been carried out by diluting the sample in an organic solvent such as n-hexane, ether-ethanol, methanol or toluene.

Continuous methods proposed for the direct analysis of olive oil can be divided in two broad categories, those developed for the determination of global parameters (e.g. total free fatty acids, total polyphenols) by using UV-Vis detection and those involving simultaneous discrimination between structurally related compounds by gas chromatography. The most relevant aspects of these determination are summarised in Table I.

The first applications dealing with the automatic determination of characteristic parameters in olive oil

Table I

Applications of the Pretreatment Module Coupled to a Spectrophotometer and a Gas Chromatograph for the Direct Analysis of Olive Oil Samples

\begin{tabular}{|c|c|c|c|c|c|c|c|}
\hline Analyte & solvent/reaction & detection & detection limit & $\begin{array}{r}\text { RSD } \\
(\%)\end{array}$ & $\begin{array}{l}\text { sampling } \\
\text { frequency } \\
\left(\mathrm{h}^{-1}\right)\end{array}$ & comments & reference \\
\hline FFA & ether-ethanol/titration & $\begin{array}{l}\text { photometry } \\
(\lambda=560 \mathrm{~nm})\end{array}$ & $<0.15 \%$ & $1.5-2.5$ & 60 & $\begin{array}{l}\text { A computer program allows direct results in } \\
\text { degree of acidity }\end{array}$ & $\begin{array}{l}\text { Linares et al. } \\
(1989)\end{array}$ \\
\hline FFA & toluene/com plexation & $\begin{array}{l}\text { photometry } \\
(\lambda=716 \mathrm{~nm})\end{array}$ & $0.02-0.05 \%$ & $4.9-7.8$ & 12 & $\begin{array}{l}\text { Similar results to that provided by a titration } \\
\text { method }\end{array}$ & Zhi et al. (1996) \\
\hline polyphenols & $\mathrm{n}$-hexane/redox & $\begin{array}{l}\text { photometry } \\
(\lambda=750 \mathrm{~nm})\end{array}$ & $<100 \mu \mathrm{g} / \mathrm{mL}$ & $2.8-4.5$ & 28 & $\begin{array}{l}\text { Liquid-liquid extraction without phase } \\
\text { separation }\end{array}$ & $\begin{array}{l}\text { García-Mesa et al. } \\
(1990)\end{array}$ \\
\hline bitterness & methanol-water & $\begin{array}{l}\text { photometry } \\
(\lambda=225 \mathrm{~nm})\end{array}$ & - & 3.2 & 12 & Matrix is retained on a $C_{18}$ packed colum $n$ & $\begin{array}{l}\text { García-Mesa et } \\
\text { al. (1992 a) }\end{array}$ \\
\hline hydroperoxides & $n$-hexane/redox & $\begin{array}{l}\text { photometry } \\
(\lambda=360,420 \mathrm{~nm})\end{array}$ & $<3.5 \mathrm{meq} / \mathrm{Kg}$ & $0.7-2.4$ & 15 & Automation of the Official European Method & $\begin{array}{l}\text { García-Mesa et al. } \\
\text { (1993 a) }\end{array}$ \\
\hline hydroperoxides & butanol-methanol/redox & $\begin{array}{l}\text { photometry } \\
(\lambda=555 \mathrm{~nm})\end{array}$ & $50 \mu \mathrm{eq} / \mathrm{Kg}$ & $1.6-2.8$ & 60 & $\begin{array}{l}\text { Peroxide value as a lower limit of the actual } \\
\text { peroxide content }\end{array}$ & $\begin{array}{l}\text { Tian and } \\
\text { Dasgupta (1999) }\end{array}$ \\
\hline triglycerides & $\mathrm{n}$-hexane/derivatisation & GC/FID & $0.5-0.9 \mu \mathrm{g} / \mathrm{mL}$ & $2.1-3.4$ & - & $\begin{array}{l}\text { On-line coupling of FI system to GC. No prior } \\
\text { saponification was necessary }\end{array}$ & $\begin{array}{l}\text { Ballesteros et al. } \\
(1993)\end{array}$ \\
\hline $\begin{array}{l}\text { cholesterol and } \\
\text { tocopherols }\end{array}$ & $n$-hexane & GC/FID & $0.5-0.8 \mu \mathrm{g} / \mathrm{mL}$ & 1.9-3.1 & - & $\begin{array}{l}\text { Triglycerides were derivatised to increase their } \\
\text { volatility }\end{array}$ & $\begin{array}{l}\text { Ballesteros et al. } \\
(1996)\end{array}$ \\
\hline sterols & $\mathrm{n}$-hexane/adsorption & GC/FID & $40-80 \mu \mathrm{g} / 100 \mathrm{~g}$ oil & $1.6-3.6$ & - & $\begin{array}{l}\text { Average recoveries of sterols added to olive oil } \\
\text { ranged from } 92 \text { to } 103 \%\end{array}$ & $\begin{array}{l}\text { Ballesteros et al. } \\
\text { (1995) }\end{array}$ \\
\hline
\end{tabular}


by spectrophotometry appeared at the beginning of the 90s. No remarkable advances have been made since then. Total free fatty acids (FFA) is one of the major parameter in establishing the quality of an oil and it is often determined in every batch of base vegetable prior to acceptance; the simplest configuration uses the same reagents as it standard counterpart (Linares et al. 1989). As can be seen in Figure 1, the oil sample was mixed with an ether-ethanol stream into the FI system and $130 \mu \mathrm{L}$ of the mixture titrated with potassium hydroxide and phenolphthalein as indicator (reagent); the baseline was established with the titrant at $560 \mathrm{~nm}$ and the decrease in absorbance as consequence of indicator decolorisation was related to the free acidity in oils. The method reduces the amounts of both, typical reagents and sample $(2 \mathrm{~mL}$ of reagent and $150 \mathrm{mg}$ of sample versus $10-20 \mathrm{~mL}$ of reagent and $5-20 \mathrm{~g}$ of sample in the conventional method) with a high sampling frequency $\left(60 \mathrm{~h}^{-1}\right)$. A slightly modified method for the determination of FFA has also been proposed (Norous et al. 1997). Automatic liquid-liquid extraction has also been used for the determination of total FFAs by using the reaction between the $\mathrm{Cu}$ (II)-pyridine complex and the FFAs, yielding a new complex which was extracted into an organic phase (Zhi, et al., 1996). Continuous monitoring of the organic phase at $716 \mathrm{~nm}$ by means of a photometer located in the middle of the injection loop provided a multi-peak absorbance-time profile representing multiple reversals in the dynamic extraction process. Total FFAs can thus be determined in contents from 0.03 to $1.5 \%$ for oleic acid in toluene-diluted olive oil samples.

The determination of the polyphenols content in olive oil is of great interest because of the natural activity of these compounds as antioxidants; in addition, olive oils with high polyphenols content possess a better sensorial quality. The conventional method for the determination of polyphenols in oil is slow and tedious ( $2 \mathrm{~h}$ per analysis). The flow configuration proposed (García-Mesa et al. 1990) is

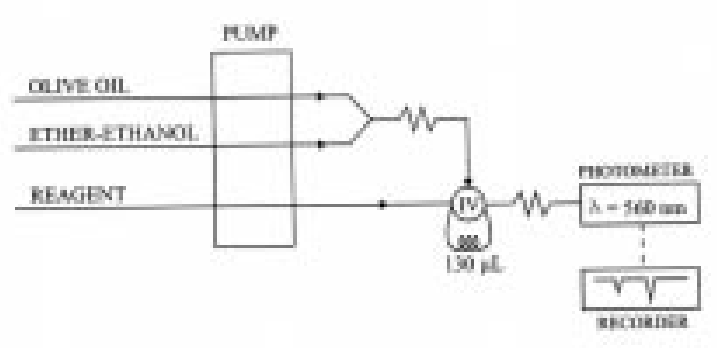

Figure 1

Manifold used for the determination of total free fatty acids in olive oil. The sample was diluted prior injection. IV, injection valve. For details, see text. based on the reaction of polyphenols with the Folin-Ciocalteu reagent. For this purposes, oil samples diluted in n-hexane were mixed with this reagent in a basic medium. The analytes were extracted into the aqueous phase by a liquid-liquid extraction system with iterative change of the flow direction, in the range over which polyphenols normally occur in these samples $(100-900 \mu \mathrm{g} / \mathrm{mL})$ with better precision and sampling frequency than that provided by the conventional method. Solid-liquid extraction is a well established alternative to liquid-liquid extraction as it circumvents some of the problems posed by the latter by using smaller volumes of toxic solvents and simplifying sample preparation. An automated version of an existing batch method for the determination of bitterness in virgin olive oil was developed (García-Mesa et al. 1992 a). The bitterness of virgin olive oil is complex; bitter oils are usually obtained from unripe olives and although these oils are rejected for consumption as such, they are highly resistant to rancidity, so they are often added to other oils of low bitterness. The UV absorption spectra of aqueous alcoholic extracts of bitter oils shows a sharp maximum at $225 \mathrm{~nm}$ and a smaller maximum at $278 \mathrm{~nm}$ being the measurements normally done at $225 \mathrm{~nm}$. The automatic method is based on FI principles and was implemented by coupling a minicolumn packed with $\mathrm{C}_{18}$-bonded silica into the loop of an injection valve for sample matrix retention, being the eluted analytes monitored by UV spectrometry; the retained matrix was further eluted in the opposite direction to detection, so it never reached the detector. The automatic method clearly surpasses its manual counterpart in terms of solvents savings, analysis time (5 vs. 50 min.) and labour requirements. The determination of the hydroperoxide value is a common practice in olive oil laboratories as this parameter, together with the UV absorbance, is used to evaluate the degree of oxidation of oils. Two FI methods have been developed for the determination of this global parameter in olive oil. The first one (García-Mesa et al. 1993 a) is based in the redox reaction of peroxides in acetic acid medium with sodium iodide and monitoring the ion $\mathrm{I}_{3}{ }^{-}$at different wavelengths. The other (Tian and Dasgupta 1999) is also based on a redox reaction between the hydroperoxides and $\mathrm{Fe}$ (II), followed by the photometric detection of $\mathrm{Fe}$ (III) as the thiocyanate complex. Taking into account its lower reaction time (30 s), the method exhibits a higher sample throughput.

Edible vegetable oils and animal fats consist mainly of triglycerides, which are responsible for their nutritional and physicochemical properties. Sterols and tocopherols belong to the group of nutritionally significant lipids that more and more often have to be routinely determined in oils. The direct gas chromatographic (GC) analysis of triglycerides is 
exceedingly difficult because of the low volatility of these compounds; thus, liquid chromatography has been proposed for the direct determination of triglycerides. The fatty acid composition of olive oil is still used by lipid analysts for the determination of adulteration; their direct determination by GC requires derivatisation of fatty acids to esters. An automatic continuous flow system has been developed for the direct analysis of olive oil. The manifold used is depicted in Figure 2; 10-120 mg of oil plus internal standard in $100 \mathrm{~mL}$ of $n$-hexane was aspirated as sample into the analyser and the fatty acids in oil were on-line methylated with acetyl chloride in methanol, followed by continuous transesterification of the triglycerides. A water stream was then inserted to effect separation of excess reagents and residual reaction products. The n-hexane phase from the membrane separator containing the fatty acid methyl esters (FAME) was introduced $(5 \mu \mathrm{L})$ via a high-pressure injection valve into the injection port of a gas chromatograph (Ballesteros et al. 1993). Comparison of this transesterification method with the manual one involving saponification with methanolic sodium hydroxide and esterification with boron trifluoride in methanol (Official Method of Analysis) revealed good agreement between the results provided by olive oils. By using an analyser similar to that depicted in Figure 2 , cholesterol, $\alpha$-tocopherol and $\alpha$-tocopheryl acetate can be determined in edible oils without derivatization (Ballesteros et al. 1996). Interferences from lipid material were avoided by transesterification of triglycerides with potassium methylate in methanol. A spiked olive oil sample containing the analytes in n-hexane was introduced into the system and merged with the derivatising reagent; triglycerides were transesterified in a heated reactor. The extract from the membrane separator filled the loop of the injection valve that acts as interface between the pre-treatment module and the instrument. Qualitative and quantitative analysis of sterols is often used for identifying lipid mixtures when adulteration is suspected; olive oil is occasionally adulterated for economic gain. Methods involving GC are the most widely used for the determination of sterols. An analyser based on flow configurations was developed for the determination of sterols in edible oils. (Ballesteros et al. 1995). The oil sample in $\mathrm{n}$-hexane, containing $0.6-70 \mu \mathrm{g} / \mathrm{mL}$ of sterols, was introduced into a silica gel minicolumn which permits the isolation of sterols together with $25 \%$ of triglycerides, being all compounds eluted with methanol. Transesterification of triglycerides was carried out similarly as depicted in Figure 2 by using acetyl chloride in n-hexane as extractant. Two types of adulteration of olive oil were studied from vegetable and animal oils. The automated method surpasses the Official Method for the determination

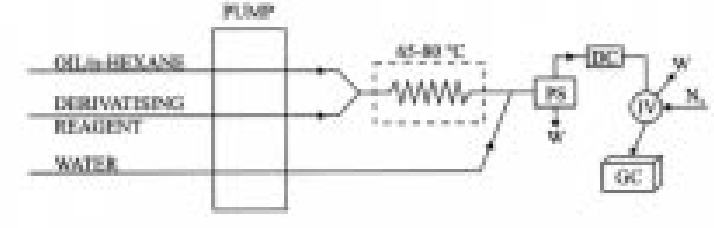

Figure 2

Schematic diagram of the system used for continuous derivatisation of triglycerides and determination of fatty acids methyl esters. PS, phase separator; DC, dessicating column;

IV, injection valve; W, waste; GC, gas chromatograph.

of sterols in oil as regards sample manipulation, which results in increased precision and sampling frequency (only limited by the oven programme temperature of the chromatograph). Also, the pretreatment module coupled on-line to gas chromatograph permits the isolation/preconcentration of analytes and the transesterification of triglycerides in a closed system without the need to handle any toxic derivatizing reagent and with minimal costs since the organic solvents and reagents are consumed very sparingly.

Full automation of the steps involved in an analytical process is difficult using an alternative different from a robotic station as the robot allows the implementation of unitary operations such as weighing and evaporation, where continuous methods failed. However, each approach has its peculiar advantages as continuous methods provide higher throughput while the robot can work unattended. In the field of edible oil analysis, some analytical determinations such as acidity, bitterness or total polyphenols, have been solved by developing automated method based on both alternatives. Total polyphenols in olive oil can be determined by implementing and on-line robotic/FI configuration (García-Mesa et al. 1993 b), where the method previously developed using FI alone (García-Mesa et al. 1990) was included as a peripheric of the robotic system. The robot performs the following unitary operations: (i) preparation of standard solutions for calibration of the FI system; (ii) isolation of the polyphenols of the olive oil sample, which involves weighing, addition of reagents, mixing, aspiration of the heavier phase, re-extraction of the sample and mixing both extract; and (iii) introduction of the extract into the $\mathrm{FI}$ system for reaction and spectrophotometric monitoring of the transient signal. The comparison of the analytical features provided by the manual, $\mathrm{FI}$ and robot- $\mathrm{Fl}$ methods resulted in excellent agreement except for the precision, which was higher for the robot-FI method (ca. 1.6\%) and the analysis time, which was lower using the FI alternative. The automation (in both 
alternatives dramatically reduces the sample amount (from $10-30 \mathrm{~g}$ to $1 \mathrm{~g}$ ) and so the reagent consumption. The determination of bitterness in virgin olive oil by using a robotic station (García-Mesa et al. 1992 b) is based on separation of the analytes by sorbent extraction (Bond Elut LRC $\mathrm{C}_{18}$ ), followed by overall photometric quantification of these compounds at $225 \mathrm{~nm}$. Although the FI approach (García-Mesa et al. 1992 a) offers two clear advantages over the robotic method namely: a higher sample throughput $\left(12 \mathrm{~h}^{-1}\right.$ versus $\left.5 \mathrm{~h}^{-1}\right)$ and lower equipment and disposable consumption costs, the robot can work unattended through a working day (seven days a week, twelve months a year). The simplicity of the operations involved in the robotic method makes the use of an inexpensive and simpler commercially available robotic system suitable for the implementation of the proposed method. A gravimetric method for the determination of the oxidative stability of olive oil based on the use of a robotic station has been proposed as an alternative to the well-established Rancimat method (García-Mesa 1993 c), which involves an endless series of weighings prone to personal errors. The robotic system monitors the weight changes of heated samples, being its main advantages over the well-established counterparts the full automation of the process and a much higher sample throughput (150 samples/batch), which amply offset the higher initial cost of the equipment required. Finally, a fully robotic method for the determination of acidity in olive oil has been proposed (Velasco-Arjona and Luque de Castro 1998), which is based on $\mathrm{pH}$ measurements on an emulsion of the oil sample in a suitable reagent $(0.2 \mathrm{M}$ triethanolamine $+0.02 \mathrm{M}$ $\mathrm{KNO}_{3}$ in a 1:1 isopropanol/water mixture, $\mathrm{pH} 11.3$ ). The robot performs the weighing of the sample, adds the reagents, emulsifies both and measures the $\mathrm{pH}$ of the emulsion, providing a sample rate of $15 \mathrm{~h}^{-1}$ with an acceptable precision.

\section{ACKNOWLEDGEMENT}

This work was supported by Grant CAO00-004 from Consejería de Agricultura y Pesca, Junta de Andalucía.

\section{REFERENCES}

Angerosa, F., Di Giacinto, L., Vito, R., Cumitini, S. (1996) Sensory evaluation of virgin oil by artificial neural network processing of dynamic headspace gas-chromatographic data. J. Sci. Food Agric., 72, 323-328.

Ballesteros, E., Gallego, M., Valcárcel, M. (1993) Automatic method for on-line preparation of fatty acids methyl esters from olive oil and other types of oil prior to their gas chromatographic determination. Anal. Chim. Acta, 282, 581-588.
Ballesteros, E., Gallego, M., Valcárcel, M. (1995) Simultaneous determination of sterols in edible oils by use of a continuous separation module coupled to a gas chromatograph. Anal. Chim. Acta, 308, 253-260

Ballesteros, E., Gallego, M., Valcárcel, M. (1996) Gas chromatographic determination of cholesterol and tocopherols in edible oils and fats with automatic removal of interfering tryglycerides. J. Chromatogr. A., 719, 221-227.

García-Mesa, J.A., Linares, P., Luque de Castro, M.D., Valcárcel, M. (1990) Direct automatic determination of polyphenols in olive oils in the aqueous phase of a flow-injection liquid-liquid extraction system without phase separation. Anal. Chim. Acta, 235, 441-444.

García-Mesa, J.A., Luque de Castro, M.D., Valcárcel, M. (1992 a) Direct automatic determination of bitterness in virgin olive oil by use of a flow injection-sorbent extraction system. Anal. Chim. Acta, 261, 367-374.

García-Mesa, J.A., Luque de Castro, M.D., Valcárcel, M. (1992 b) Determination of bitterness in virgin olive oil by using a robotic station. $L R A, 5,29-32$.

García-Mesa, J.A., Luque de Castro, M.D., Valcárcel, M. (1993 a) Automated determination of peroxides in olive oil by flow injection. Analyst, 118, 891-893.

García-Mesa, J.A., Luque de Castro, M.D., Valcárcel, M. (1993 b) Coupled robot-flow injection analysis system for fully automated determination of total polyphenols in olive oil. Anal. Chem., 65, 3540-3542.

García-Mesa, J.A., Luque de Castro, M.D., Valcárcel, M. (1993 c) Determination of the oxidative stability of olive oil by use of a robotic station. Talanta, 40, 1595-1600.

García-Pinto, C., Fernández-Laespada, M.E., PerezPavón, J.L., Moreno-Cordero, B. (2001) Electronic olfactometry. A new tool in analytical chemistry. Quim. Anal., 20, 3-11.

Gardner, J.W., Bartlett, P.N. (1999). Electronic noses. Principles and applications, Oxford University Press. Inc, New York.

Guadarrama, A., Rodríguez-Méndez, M.L., de Saja, J.A., Rios, J.L., Olías, J.M. (2000) Array of sensors based on conducting polymers for the quality control of the aroma of the virgin olive oil. Sens. Actuators B, 69, 276-282.

Linares, P., Luque de Castro, M.D., Valcárcel, M. (1989) Direct automatic determination of free acidity in oils by flow-injection analysis. Anal. Chim. Acta, 225, 431-436.

Meng, C.K., Wylie, P.L. (1999) Using a mass selective chemical sensor to help identify unknowns. Hewlett-Packard Application Note 228-410.

Morales, M.T., Aparicio, R., Ríos, J.J. (1994) Dynamic headspace gas-chromatographic method for determining volatiles in virgin olive oil. J. Chromatogr. A, 668, 455- 462 .

Nerin, C., Gancedo, P., Cacho, J. (1993) Determination of styrene in olive oil by coevaporation, cold trap and GC-MS-SIM. J. Agric. Food Chem., 41, 2003-2005.

Norman, K.N.T. (1991) Determination of tetrachloroethylene in olive oil by automated headspace gas chromatography. Food Addit. Contam., 8, 513-516.

Nouros, P.G., Georgiou, C.A., Polissiou, M.G. (1997) Automated flow-injection -spectrophotometric non-aqueous titrimetric determination of the free fatty acid content of olive oil. Anal. Chim. Acta, 351, 291-297.

Overton, S. V., Manura, J.J. (1995) Analysis of volatile organics in cooking oils by thermal desorption gas chromatography-mass spectrometry. J. Agric. Food Chem., 43, 1314-1320. 
Stella, R., Barisci, J.N., Serra, G., Wallace, G.G., De Rossi, D. (2000) Characterisation of olive oil by an electronic nose based on conducting polymer sensors. Sens. Actuators B, 63, 1-9.

Tian, K., Dasgupta, P.K. (1999) Automated measurement of lipid hydroperoxides in oil and fat samples by flow injection photometry. Anal. Chem., 71, 2053-2058.

Valcárcel, M., Luque de Castro, M.D., Tena, M.T. (1993) Preliminary operations: A pending goal of today's analytical chemistry. Anal. Proc., 30, 276-279.

Valcárcel, M., Cárdenas, S., Gallego, M. (1999) Sample screening systems in analytical chemistry. Trends Anal. Chem., 18, 685-694.
Valcárcel, M., Cárdenas, S., Gallego, M. (2000) Qualitative analysis revisited. Crit. Rev. Anal. Chem., 30, 345-361.

Velasco-Arjona, A., Luque de Castro, M.D. (1998) A fully robotic method for the determination of acid values in olive oil without titration. J. Am. Oil. Chem. Soc., 75, 1849-1853.

Zhi, Z., Ríos, A., Valcárcel, M. (1996) An automated flow-reversal injection/liquid-liquid extraction approach to the direct determination of total free fatty acids in olive oils. Anal. Chim. Acta, 318, 187-194. 